\title{
Improving Growth and Productivity of Pomegranate Fruit Trees Planted On Sandy Dunes Slopes at Baloza District (N. Sinai) Using Different Methods of Drip Irrigation, Organic Fertilization, and Soil Mulching
}

\author{
Mahmoud I. El -Desouky and Sheren A. Abd El- Hamied \\ Desert Research Center, Cairo, Egypt
}

\begin{abstract}
This study was carried out during the two successive seasons of 2012 and 2013 at Baloza Experimental Station, Desert Research Center, North Sinai Governorate, Egypt, on Manfalouty pomegranate (cv.) shrubs planted on the slops of sandy dune. All the area subjected to the same agriculture practice. The study aimed to plan such model of farm management for planting the sand dune slops. The experimental work depended on modifying some traditional methods of irrigation, fertilization and protecting soil surface from erosion and water loss. Two irrigation systems (surface and sub surface drip irrigation) with organic fertilization (control 1 (without), compost and goat manure) and soil mulching (control 2 (without), plastic sheets and rice straw) were the main treatments applied to achieve the aim of the work and evaluate the improvement or that highly economic value fruit. The obtained results cleared that, sub surface drip irrigation was better than surface drip irrigation system. In addition, using plastic sheet as a mulching and compost as organic fertilization under sub surface drip irrigation gave the high leaf area, leaf chlorophyll, number of leaves/shoot, shoot length, number of followers / shoot, fruit set \%, total yield, fruit length, fruit diameter, fruit weight, grains weight, TSS, total sugar content and vitamin C in both seasons. On the other side, surface drip irrigation without any organic fertilization and soil mulching gave the highest total acidity.
\end{abstract}

Key words: irrigation systems, Manfalouty cultivar, organic fertilization, pomegranate, sand dune fixation, soil conditioners, soil mulching.

\section{Introduction}

Pomegranate (Punica granatum L.) is one of the family Punicaceae plants and is mainly belongs to semi-arid mild-temperate to subtropical climates. Pomegranate orchards are now grown in many regions of the world, particularly in the Mediterranean Basin, where high quality fruits are obtained [Stover and Mercure, (2007) and Holland et al., (2009)]. Manfalouty is considered as one of the most important pomegranate cultivar grown successfully in Egypt.

Sand dunes are of the problems which seriously affecting the agricultural activities even the area it self. In Egypt sand dunes cover about $16 \%$ of the whole country. Sandy dunes slopes could be cultivated using some materials (such as mulching materials, and some soil conditioners) help protecting from water loss. Morphologically sand dunes are subdivided into sand seas (ergs), isolated dunes and dune fields and sandy plains. In Egypt, sand encroachment causes hazards to farmlands, highways, population centre and other infrastructures (Misak and Draz 1997).

Developed irrigation systems are very important for saving irrigation water which is the most limiting and most precious resources for agriculture today (Helweg, 1989). Drip irrigation systems are having an important priority in new the reclaimed area. Surface and subsurface drip irrigation methods can play a significant role in overcoming the scarcity of water mostly in water shortage areas. Talat et al (2012). The drip irrigation is reported to perform better in light sandy soils and water scarcity regions under deep ground water table conditions, but the performance of the drip irrigation should be tested under adverse conditions of shallow water table and heavy soils. Subsurface drip irrigation allows uniform soil moisture, minimize the evaporative loss and delivery water directly to the plant root zone which increases use efficiency and yield (Douh et al., 2013). In addition, Talat et al., (2012) found that yield of date palms was increased by 45 and $48 \%$ under sub surface irrigation as compared with surface drip irrigation. Bryla, et al., (2003) studied the effects of furrow, microjet, surface drip, and sub surface drip irrigation on vegetative growth and early production of 'Crimson Lady' peach [Prunus persica (L.) Batsch] trees. In addition, he stated that overall performance showed that trees irrigated by surface and subsurface drip were significantly larger and produced higher yields, than trees irrigated by microjets.

Desert soils suffer mainly from the shortage in water. So, several soil management practices are adopted to restrict water losses and to maximize the output of limited water resources. One of the most important and effective measures for controlling soil water and improving thermal regime is soil mulching to 
protect the soil surface. Also, it is frequently applied around plants to conserve soil water, modify the soil environment, reduce heat losses in winter and enhance plant growth. (Monteith, 1973). Mulch is a protective cover placed over the soil surface. Mulch can play an important role for sustainable fruit production. Beneficial aspects of plastic mulch include conservation of moisture, controls weeds and moderate soil temperature for better root growth and higher crop yield [Ramakrishna, et al (2006), and Sudadi et al,. (2007)]. Plastic mulches reduce the amount of water lost from the soil due to evaporation. This means less water will be needed for irrigation. Plastic mulches also aid in evenly distributing moisture to the soil which reduces plant stress. Plastic mulches prevent sunlight from reaching the soil so it can inhibit most annual and perennial weeds, (Tarara, 2000). Albert et al. (2010) reported that mulching have significantly influence on $\mathrm{pH}$ and nutrient content. The highest fruit yield of Pineapple (Ananas comosus. L. Merr) was recorded with polythene treatment. Janaki and Hkmsk (2009) and El Mardi et al., (2007) mentioned that mulching the soil with plastic cover gave the highest fruit length, diameter and yield in date palm (Khalas). EI-Kosary, et al., (2009) added that plastic mulch had enhanced the fruit characteristics of Zaghloul date palm.

Drip irrigation systems was found to result in 30 to $70 \%$ water savings in various orchard crops and vegetables along with 10 to $60 \%$ increases in yield as compared to conventional methods of irrigation. It is prudent to make efficient use of water and bring more area under irrigation through available water resources. This can be achieved by introducing advanced methods of irrigation and improved water management practices (Zaman et al., 2001). Drip irrigation in combination with mulch is one of the best irrigation methods, which can improve the water management practice significantly. Surface mulches have been used to improve soil water retention, reduce soil temperature and reduce wind velocity at the soil surface and arid lands (Kay, 1978; Jalota and Prihar, 1998). Surface mulches can also improve water penetration by impeding runoff and protecting the soil from raindrop splash and reducing soil crusting (Munshower, 1994). In addition, Tiwari et al., (2014) recorded that plastic mulch and drip irrigation increase yield in Sapota (Achras zapota) as compared with ring basin irrigation.

Growing plants in the newly reclaimed soils is faced by various problems, such as cultivars, fertilization, low amounts of available nutrients and low organic matter content as well as poor hydrophilic, chemical and biological properties. The best means of maintaining soil fertility and productivity could be through periodic addition of organic manures such as compost and goat manure, which can secure sustainable nutrients as the needs of the plants for growth and thrive. The slow release of nutrients from organic fertilizers and trace elements content increase the suitability of organic fertilizers as a sandy soil amendments which improve soil structure and fertility, controlling soil ph and provide the plants with mineral needs, that is not mostly found in most chemical formulations. In addition, organic manures have been shown largely improve in soil physical conditions such as moisture retention, aggregate soil stability capacity and crop performance. (Hati et al 2006). Manure and compost increase soil organic matter, improve soil structure, increase the water-holding capacity of coarse-textured sandy soils, improve drainage in fine-textured clay soils, provide a source of slow release nutrients, reduce wind and water erosion, and promotes growth of earthworms and other beneficial soil organisms. Most vegetable crops return small amounts of crop residues to the soil, so manure, compost, and other organic amendments help maintain soil organic matter levels. Organic manures are able to enhance soil microbial activity and improve the enzyme activity by increasing soil microbial biomass (Ren et al., 1996; Sun et al., 2003; Lu et al., 2005). Compost is one of the most effective ways of applying organic matter to soils and improving organic carbon levels. Composts are used to increase crop productivity and yields, and their use is usually associated with improving soil structure and enhancing soil fertility, increasing soil microbial populations and activity and the improvement of moisture-holding capacity of the soil (Arancon, et al., 2004).

As mentioned before, sand dunes are one of the most negative environmental phenomena that facing all the economic activities including agriculture even domestication it self. Moreover, planting sand dunes is one of the most important ways for sand dune fixation. On the other hand, planting sand dunes slopes with fruit trees is one of untraditional farming systems especially under the Egyptian environments. So, this trail is aiming to plan such model of farm management for planting fruit orchard on sand dunes slopes the experiment work depended on modifying the traditional method of irrigation and fertilization by protecting the soil surface from erosion, and save most of sub surface moisture from loss. The final gall is to overcome the early deterioration of pomegranate orchard planted on a sand dune slope, and improve growth and productivity of the pants.

\section{Materials and Methods}

This study was conducted during the two successive seasons of 2012 and 2013 at Baloza Experimental Station, Desert Research Center, North Sinai Governorate, Egypt, on pomegranate shrubs (Punica granatum L.) of Manfalouty cultivar. Trees under investigation were grown in a sandy soil (Table 1). Planting space was $4 \times 4$ $\mathrm{m}$ apart. The experiment was designed as spilt spilt plot design. Three replicates were used for each treatment and every replicate was represented by three trees with $(2 \times 3 \times 3)$ in a factorial arrangement of treatments with the following factors and levels: main factor irrigation systems (sub surface drip or surface drip), sub main organic 
fertilization (control 1 (without), compost (10 kg/tree) and goat manure (10 kg/ tree) (Table 2) and sub sub main mulching (control 2 (without), plastic sheet and rice straw).

Table 1: Some physical and chemical properties of the experimental soil.

\begin{tabular}{|c|c|c|c|c|c|c|c|c|c|c|c|c|c|c|}
\hline \multicolumn{3}{|c|}{$\begin{array}{l}\text { Particle size } \\
\text { distribution }\end{array}$} & \multirow[t]{2}{*}{$\begin{array}{l}\text { Texture } \\
\text { soil }\end{array}$} & \multirow{2}{*}{$\begin{array}{l}\text { Ec } \\
\text { dsm }^{-1}\end{array}$} & \multirow[t]{2}{*}{$\mathbf{p H}$} & \multicolumn{5}{|c|}{$\begin{array}{l}\text { Available nutrients } \\
\text { (Cation) }\end{array}$} & \multicolumn{4}{|c|}{$\begin{array}{l}\text { Available nutrients } \\
\text { ( Anion) }\end{array}$} \\
\hline Sand & Silt & Clay & & & & $\begin{array}{l}\mathrm{Na} \\
\%\end{array}$ & $\begin{array}{l}\mathbf{P} \\
\%\end{array}$ & $\begin{array}{c}\mathbf{K} \\
\%\end{array}$ & $\begin{array}{l}\mathrm{Ca} \\
\mathrm{meg} / \mathrm{l}\end{array}$ & $\begin{array}{l}\mathrm{Mg} \\
\mathrm{meg} / \mathrm{l}\end{array}$ & $\mathrm{CO3}$ & $\begin{array}{c}\mathrm{HCO3} \\
\mathrm{meg} / \mathrm{l}\end{array}$ & $\mathrm{Cl}^{-}$ & $\mathrm{So}_{4}^{--}$ \\
\hline 90 & 5 & 5 & sandy & 1.37 & 8.20 & 4.78 & 0.42 & 0.54 & 3.65 & 4.40 & - & 3.85 & 3.3 & 6.5 \\
\hline
\end{tabular}

Table 2: Some chemical properties of goat manure and compost.

\begin{tabular}{|l|c|c|}
\hline properties & Goat manure & Compost EI Neel \\
\hline Organic matter \% & 68.2 & 30.70 \\
\hline Total N \% & 2.56 & 1.66 \\
\hline Total P \% & 2.40 & 0.44 \\
\hline Total K \% & 2.27 & 1.56 \\
\hline C: N & 19 & 18.70 \\
\hline
\end{tabular}

The following parameters were measured for both seasons:

1-Leaf Area $\left(\mathbf{c m}^{2}\right)$ : Was determined by using the leaf area meter CL203.

\section{2-Average total chlorophyll content}

Total chlorophyll content (in fresh leaves) was measured on the third leaf from the base at the end of August in field by using Minolta meter SPAD-502.

\section{3-Number of leaves per shoot}

Leaves developed on the new shoots were also counted at the end of growing season in September.

\section{4-Length of the new developed shoots $(\mathrm{cm})$}

Ten of one year old shoots was collected from around the canopy representing the four main directions were tagged for measuring new developed shoots length at the end of growing season in September.

\section{5- Number of followers / shoot and fruit set \%}

Number of followers / shoot was counted at balloon stage and fruit set $\%$ was counted two weeks after full bloom. Fruit set $\%$ was calculated according to the formula: Fruit set $\%=$ number of set fruits $\div$ total number of flowers (balloon stage) x 100

\section{6- Fruit parameters (fruit physical characteristics)}

Fruits sample was taken at the harvest time to be used for determining the physical properties (i.e., fruit length $(\mathrm{cm})$, fruit diameter $(\mathrm{cm})$, fruit weight $(\mathrm{g})$, grains weight $(\mathrm{g})$, juice percentage and peel thickness $(\mathrm{mm})$.

\section{7- Fruit quality (fruit chemical characteristics)}

a sample of 8 ripe fruits of each tree were taken at the harvest time to be used for determining the chemical properties i.e., (T.S.S.\%) that measured by using a hand refractometer and the acidity \% as citric acid content using fresh juice with titration against $0.1 \mathrm{Na} \mathrm{OH}$. The total sugars content, and content of vitamin $\mathrm{C}$ according to A.O.A.C (1985) were determined.

\section{Statistical analysis}

The data were subjected to analysis of variance and Duncan's multiple range tests was used to differentiate means as described by Duncan (1955).

\section{Results and Discussion}

\section{Leaf area, leaf chlorophyll, number of leaves / shoot and shoot length}

Results in Table (3 and 4) showed that leaf area, leaf chlorophyll, number of leaves / shoot and shoot length was significantly affected by all treatments in both seasons. It is obvious that subsurface drip irrigation system increased leaf area, leaf chlorophyll, number of leaves / shoots and shoots length in both seasons as compared with surface drip irrigation. In addition, adding compost as an organic fertilization gave the highest leaf area, leaf chlorophyll, number of leaves /shoot and shoot length in both seasons. Furthermore, mulching the soil with plastic cover gave the best leaf area, leaf chlorophyll, number of leaves / shoots and shoots length as compared with control. 
On the other hand, the interactions between irrigation system and organic fertilization show that compost under sub surface drip irrigation gave the highest leaf area (4.91 in first season and $5.29 \mathrm{~cm}^{2}$ in second seasons), leaf chlorophyll (54.56 in first season and 55.05\% in second seasons), number of leaves / shoot (27.62 in first season and 27.57 in second seasons) and shoot length (27.78 in first season and $27.79 \mathrm{~cm}$ in second seasons respectively).

In addition, the interaction between plastic cover and sub surface drip irrigation system was the best than surface drip irrigation without mulching in both seasons.

However, the interactions between compost and mulching with plastic cover gave the highest value in these parameters compared with control in both seasons.

Moreover the interactions between irrigation system, organic fertilization and soil mulching clear that added compost and mulching the soil with plastic cover under sub surface drip irrigation gave the best leaf area $\left(5.60 \mathrm{~cm}^{2}\right.$ in first season and $6.09 \mathrm{~cm}^{2}$ in second seasons), leaf chlorophyll $(61.45$ in first season and $62.12 \%$ in second seasons), number of leaves / shoot (30.19 in first season and 30.20 in second seasons) and shoot length (30.41 in first season and $30.33 \mathrm{~cm}$ in second seasons). On the other hand, surface drip irrigation without fertilization and mulching gave the less leaf area, leaf chlorophyll, number of leaves / shoot and shoot length in both seasons.

These results are in harmony with those obtained by Bryla, et al (2003) who found that sub surface drip irrigation improve vegetative growth of newly planted 'Crimson Lady' peach [Prunus persica (L.) Batsch] trees. Tiwari et al., (2014) recorded that plastic mulch and drip irrigation increase growth parameters in Sapota (Achras zapota) as compared with ring basin irrigation. In addition, Safar and Ahmed (2012) found that adding compost gave the highest leaf area and shoot length of Picual olive trees.

Table 3. Effect of irrigation systems, organic fertilization and soil mulching on leaf area and leaf chlorophyll of Manfalouty cv. pomegranate trees during 2012 and 2013 seasons.

\begin{tabular}{|c|c|c|c|c|c|c|}
\hline \multirow{2}{*}{\multicolumn{3}{|c|}{ Treatments }} & \multicolumn{2}{|c|}{ Leaf area $(\mathbf{c m} 2)$} & \multicolumn{2}{|c|}{ Leaf chlorophyll\% } \\
\hline & & & 2012 & 2013 & 2012 & 2013 \\
\hline \multicolumn{7}{|c|}{ Effect of drip irrigation systems } \\
\hline \multicolumn{3}{|c|}{ sub surface } & $3.95 \mathrm{a}$ & $4.09 \mathrm{a}$ & $46.88 \mathrm{a}$ & $47.15 \mathrm{a}$ \\
\hline \multicolumn{3}{|c|}{ surface } & $3.50 \mathrm{~b}$ & $3.54 \mathrm{~b}$ & $43.50 \mathrm{~b}$ & $43.70 \mathrm{~b}$ \\
\hline \multicolumn{7}{|c|}{ Effect of organic fertilization } \\
\hline \multicolumn{3}{|c|}{ control 1 (without) } & $2.85 \mathrm{c}$ & $2.89 \mathrm{c}$ & $38.68 \mathrm{c}$ & $38.77 \mathrm{c}$ \\
\hline \multicolumn{3}{|l|}{ compost } & $4.59 \mathrm{a}$ & $4.82 \mathrm{a}$ & $51.92 \mathrm{~b}$ & $52.31 \mathrm{a}$ \\
\hline \multicolumn{3}{|l|}{ goat manure } & $3.73 \mathrm{~b}$ & $3.74 \mathrm{~b}$ & $44.97 \mathrm{a}$ & $45.18 \mathrm{~b}$ \\
\hline \multicolumn{7}{|c|}{ Effect of soil mulching } \\
\hline \multicolumn{3}{|l|}{ control 2 (without) } & $2.79 \mathrm{c}$ & $2.80 \mathrm{c}$ & $38.44 \mathrm{c}$ & $38.68 \mathrm{c}$ \\
\hline \multicolumn{3}{|l|}{ plastic sheet } & $4.32 \mathrm{a}$ & $4.42 \mathrm{a}$ & $49.44 \mathrm{a}$ & $49.83 \mathrm{a}$ \\
\hline \multicolumn{3}{|l|}{ rice straw } & $4.07 \mathrm{~b}$ & $4.21 \mathrm{~b}$ & $47.68 \mathrm{~b}$ & $47.76 \mathrm{~b}$ \\
\hline \multicolumn{7}{|c|}{ Effect of drip irrigation systems and organic fertilization } \\
\hline \multicolumn{3}{|c|}{ sub surface + control1 } & $3.05 \mathrm{e}$ & $3.12 \mathrm{e}$ & $39.99 \mathrm{e}$ & $40.04 \mathrm{e}$ \\
\hline \multicolumn{3}{|c|}{ sub surface+ compost } & $4.91 \mathrm{a}$ & $5.29 \mathrm{a}$ & $54.56 \mathrm{a}$ & $55.05 \mathrm{a}$ \\
\hline \multicolumn{3}{|c|}{ sub surface + goat manure } & $3.88 \mathrm{c}$ & $3.85 \mathrm{c}$ & $46.08 \mathrm{c}$ & $46.35 \mathrm{c}$ \\
\hline \multicolumn{3}{|l|}{ surface + control1 } & $2.66 \mathrm{f}$ & $2.66 \mathrm{f}$ & $37.35 \mathrm{f}$ & $37.51 \mathrm{f}$ \\
\hline \multicolumn{3}{|l|}{ surface + compost } & $4.28 \mathrm{~b}$ & $4.34 \mathrm{~b}$ & $49.27 \mathrm{~b}$ & $49.57 \mathrm{~b}$ \\
\hline \multicolumn{3}{|c|}{ surface + goat manure } & $3.58 \mathrm{~d}$ & $3.09 \mathrm{~d}$ & $43.86 \mathrm{~d}$ & $44.01 \mathrm{~d}$ \\
\hline \multicolumn{7}{|c|}{ Effect of drip irrigation systems and soil mulching } \\
\hline \multicolumn{3}{|c|}{ sub surface + control 2} & $3.02 \mathrm{e}$ & $3.01 \mathrm{e}$ & $39.45 \mathrm{e}$ & $39.79 \mathrm{e}$ \\
\hline \multicolumn{3}{|c|}{ sub surface + plastic sheet } & $4.57 \mathrm{a}$ & $4.78 \mathrm{a}$ & $51.68 \mathrm{a}$ & $52.19 \mathrm{a}$ \\
\hline \multicolumn{3}{|c|}{ sub surface + rice straw } & $4.25 \mathrm{~b}$ & $4.48 \mathrm{~b}$ & $49.51 \mathrm{~b}$ & $49.46 \mathrm{~b}$ \\
\hline surface + control 2 & & & $2.55 \mathrm{f}$ & $2.59 \mathrm{f}$ & $37.44 \mathrm{f}$ & $37.56 \mathrm{f}$ \\
\hline surface + plastic shee & & & $4.07 \mathrm{c}$ & $4.11 \mathrm{c}$ & $47.19 \mathrm{c}$ & $47.48 \mathrm{c}$ \\
\hline surface + rice straw & & & $3.98 \mathrm{~d}$ & $3.93 \mathrm{~d}$ & $45.86 \mathrm{~d}$ & $46.05 \mathrm{~d}$ \\
\hline Effect of organic fe & ilization and soil mul & aing & & & & \\
\hline control $1+$ control 2 & & & $2.29 \mathrm{i}$ & $2.32 \mathrm{i}$ & $36.26 \mathrm{i}$ & $36.48 \mathrm{i}$ \\
\hline compost + control 2 & & & $3.26 \mathrm{f}$ & $3.32 \mathrm{f}$ & $40.23 \mathrm{f}$ & $40.58 \mathrm{f}$ \\
\hline goat manure + contr & & & $3.01 \mathrm{~g}$ & $3.04 \mathrm{~g}$ & $39.54 \mathrm{~g}$ & $39.26 \mathrm{~g}$ \\
\hline control $1+$ plastic $\mathrm{s}$ & & & $3.46 \mathrm{e}$ & $3.50 \mathrm{e}$ & $41.78 \mathrm{e}$ & $42.06 \mathrm{e}$ \\
\hline compost + plastic sh & & & $5.31 \mathrm{a}$ & $5.61 \mathrm{a}$ & $58.36 \mathrm{a}$ & $58.90 \mathrm{a}$ \\
\hline goat manure + plasti & sheet & & $5.02 \mathrm{~b}$ & $5.34 \mathrm{~b}$ & $55.60 \mathrm{~b}$ & $55.97 \mathrm{~b}$ \\
\hline control $1+$ rice straw & & & $2.62 \mathrm{~h}$ & $2.58 \mathrm{~h}$ & $37.29 \mathrm{~h}$ & $37.49 \mathrm{~h}$ \\
\hline compost + rice straw & & & $4.39 \mathrm{c}$ & $4.40 \mathrm{c}$ & $49.72 \mathrm{c}$ & $50.02 \mathrm{c}$ \\
\hline goat manure + rice $s$ & & & $4.18 \mathrm{~d}$ & $4.25 \mathrm{~d}$ & $47.91 \mathrm{~d}$ & $48.04 \mathrm{~d}$ \\
\hline Effect of drip irrig & ion systems, organic & rtilization and & ching & & & \\
\hline drip irrigation & oroanic fertilization & soil mulching & Leaf a & & Leaf cl & $11 \%$ \\
\hline systems & organic rertimzation & soil muicning & 2012 & 2013 & 2012 & 2013 \\
\hline cub curfoce & control 1 & control 2 & $2.46 \mathrm{~m}$ & $2.50 \mathrm{~m}$ & $36.52 \mathrm{pq}$ & $36.85 \mathrm{p}$ \\
\hline suo surlace & control 1 & plastic sheet & $3.55 \mathrm{~h}$ & $3.66 \mathrm{i}$ & $42.23 \mathrm{j}$ & $42.60 \mathrm{j}$ \\
\hline
\end{tabular}


Improving growth and productivity of pomegranate fruit trees planted on sandy dunes slopes at...

\begin{tabular}{|c|c|c|c|c|c|c|}
\hline & control 1 & rice straw & $3.17 \mathrm{i}$ & $3.20 \mathrm{j}$ & $41.24 \mathrm{k}$ & $40.67 \mathrm{k}$ \\
\hline & compost & control 2 & $3.92 \mathrm{~g}$ & $3.93 \mathrm{~h}$ & $44.34 \mathrm{i}$ & $44.88 \mathrm{i}$ \\
\hline & compost & plastic sheet & $5.60 \mathrm{a}$ & $6.09 a$ & $61.45 \mathrm{a}$ & $62.12 \mathrm{a}$ \\
\hline & compost & rice straw & $5.22 \mathrm{~b}$ & $5.86 \mathrm{~b}$ & $57.89 \mathrm{~b}$ & $58.14 \mathrm{~b}$ \\
\hline & goat manure & control 2 & 2.721 & $2.60 \mathrm{~m}$ & 37.48no & $37.65 \mathrm{no}$ \\
\hline & goat manure & plastic sheet & $4.56 \mathrm{e}$ & $4.58 \mathrm{e}$ & $51.36 \mathrm{e}$ & $51.84 \mathrm{e}$ \\
\hline & goat manure & rice straw & $4.35 \mathrm{f}$ & $4.38 \mathrm{f}$ & $49.40 \mathrm{f}$ & $49.57 \mathrm{f}$ \\
\hline \multirow{9}{*}{ surface } & control 1 & control 2 & $2.14 n$ & $2.14 n$ & $36.00 \mathrm{q}$ & $36.12 q$ \\
\hline & control 1 & plastic sheet & $2.97 \mathrm{jk}$ & $2.98 \mathrm{kl}$ & $38.23 \mathrm{~m}$ & $38.55 \mathrm{~m}$ \\
\hline & control 1 & rice straw & $2.85 \mathrm{kl}$ & 2.871 & $37.83 \mathrm{mn}$ & $37.85 n$ \\
\hline & compost & control 2 & $3.00 \mathrm{j}$ & $3.07 \mathrm{jk}$ & 39.231 & 39.241 \\
\hline & compost & plastic sheet & $5.01 \mathrm{c}$ & $5.12 \mathrm{c}$ & $55.27 \mathrm{c}$ & $55.67 \mathrm{c}$ \\
\hline & compost & rice straw & $4.81 \mathrm{~d}$ & $4.82 \mathrm{~d}$ & $53.32 \mathrm{~d}$ & $53.80 \mathrm{~d}$ \\
\hline & goat manure & control 2 & $2.52 \mathrm{~m}$ & $2.56 \mathrm{~m}$ & 37.09 op & 37.330 \\
\hline & goat manure & plastic sheet & $4.21 \mathrm{f}$ & $4.22 \mathrm{fg}$ & $48.07 \mathrm{~g}$ & $48.21 \mathrm{~g}$ \\
\hline & goat manure & rice straw & $4.01 \mathrm{~g}$ & $4.11 \mathrm{~g}$ & $46.42 \mathrm{~h}$ & $46.50 \mathrm{~h}$ \\
\hline
\end{tabular}

Means having the same letter (s) in each column or interaction are not significantly different at $5 \%$ level.

Table 4. Effect of irrigation systems, organic fertilization and soil mulching on number of leaves / shoot and shoot length of Manfalouty cv. pomegranate trees during 2012 and 2013 seasons.

\begin{tabular}{|c|c|c|c|c|c|c|}
\hline \multirow{2}{*}{\multicolumn{3}{|c|}{ Treatments }} & \multicolumn{2}{|c|}{ Number of leaves / shoot } & \multicolumn{2}{|c|}{ Shoot length $(\mathrm{cm})$} \\
\hline & & & 2012 & 2013 & 2012 & 2013 \\
\hline \multicolumn{7}{|c|}{ Effect of drip irrigation systems } \\
\hline \multicolumn{3}{|c|}{ sub surface } & $24.22 \mathrm{a}$ & $24.31 \mathrm{a}$ & $24.32 \mathrm{a}$ & $24.40 \mathrm{a}$ \\
\hline \multicolumn{3}{|c|}{ surface } & $22.79 b$ & $22.87 b$ & $22.85 b$ & $23.02 \mathrm{~b}$ \\
\hline \multicolumn{7}{|c|}{ Effect of organic fertilization } \\
\hline \multicolumn{3}{|c|}{ control 1 (without) } & $20.23 c$ & $20.35 \mathrm{c}$ & $20.44 c$ & $20.62 \mathrm{c}$ \\
\hline \multicolumn{3}{|l|}{ compost } & $26.90 \mathrm{a}$ & $26.95 a$ & $26.91 \mathrm{a}$ & $27.01 \mathrm{a}$ \\
\hline \multicolumn{3}{|c|}{ goat manure } & $23.39 b$ & $23.47 b$ & $23.41 b$ & $23.51 \mathrm{~b}$ \\
\hline \multicolumn{7}{|c|}{ Effect of soil mulching } \\
\hline \multicolumn{3}{|c|}{ control 2 (without ) } & $19.94 \mathrm{c}$ & $20.07 \mathrm{c}$ & $20.26 \mathrm{c}$ & $20.36 c$ \\
\hline \multicolumn{3}{|l|}{ plastic sheet } & $25.69 \mathrm{a}$ & $25.86 \mathrm{a}$ & $25.61 \mathrm{a}$ & $25.72 \mathrm{a}$ \\
\hline \multicolumn{3}{|c|}{ rice straw } & $24.89 b$ & $24.84 b$ & $24.88 b$ & $25.05 \mathrm{~b}$ \\
\hline \multicolumn{7}{|c|}{ Effect of drip irrigation systems and organic fertilization } \\
\hline \multicolumn{3}{|c|}{ sub surface + controll } & $21.10 \mathrm{e}$ & $21.25 \mathrm{e}$ & $21.25 \mathrm{e}$ & $21.43 \mathrm{e}$ \\
\hline \multicolumn{3}{|c|}{ sub surface+ compost } & $27.62 \mathrm{a}$ & $27.57 \mathrm{a}$ & $27.78 \mathrm{a}$ & $27.79 \mathrm{a}$ \\
\hline \multicolumn{3}{|c|}{ sub surface + goat manure } & $23.95 \mathrm{c}$ & $24.04 \mathrm{c}$ & $23.93 \mathrm{c}$ & $24.00 \mathrm{c}$ \\
\hline \multicolumn{3}{|c|}{ surface + control 1} & $19.35 f$ & $19.46 \mathrm{f}$ & $19.63 \mathrm{f}$ & $19.81 \mathrm{f}$ \\
\hline \multicolumn{3}{|l|}{ surface + compost } & $26.18 \mathrm{~b}$ & $26.23 \mathrm{~b}$ & $26.04 \mathrm{~b}$ & $26.24 b$ \\
\hline \multicolumn{3}{|c|}{ surface + goat manure } & $22.84 d$ & $22.91 \mathrm{~d}$ & $22.88 \mathrm{~d}$ & $23.02 d$ \\
\hline Effect of drip irrig & ion systems a & nulching & & & & \\
\hline sub surface + contro & & & $20.67 \mathrm{e}$ & $20.79 \mathrm{e}$ & $20.95 \mathrm{e}$ & $21.04 \mathrm{e}$ \\
\hline sub surface + plastic & heet & & $26.48 \mathrm{a}$ & $26.60 \mathrm{a}$ & $26.45 a$ & $26.50 \mathrm{a}$ \\
\hline sub surface + rice st & & & $25.51 \mathrm{~b}$ & $25.55 b$ & $25.53 b$ & $25.66 \mathrm{~b}$ \\
\hline surface + control 2 & & & $19.21 \mathrm{f}$ & $19.35 \mathrm{f}$ & $19.57 \mathrm{f}$ & $19.68 \mathrm{f}$ \\
\hline surface + plastic she & & & $24.90 \mathrm{c}$ & $25.12 \mathrm{c}$ & $24.76 \mathrm{c}$ & $24.94 \mathrm{c}$ \\
\hline surface + rice straw & & & $24.26 \mathrm{~d}$ & $24.14 d$ & $24.23 d$ & $24.44 d$ \\
\hline Effect of organic fe & tilization and & ching & & & & \\
\hline control $1+$ control & & & $17.46 \mathrm{i}$ & $17.58 \mathrm{i}$ & $18.20 \mathrm{i}$ & $18.35 \mathrm{i}$ \\
\hline compost + control & & & $21.90 \mathrm{f}$ & $22.10 \mathrm{f}$ & $21.85 \mathrm{f}$ & $22.13 f$ \\
\hline goat manure + cont & & & $21.31 \mathrm{~g}$ & $21.39 \mathrm{~g}$ & $21.28 \mathrm{~g}$ & $21.38 \mathrm{~g}$ \\
\hline control $1+$ plastic & & & $22.75 \mathrm{e}$ & $22.84 \mathrm{e}$ & $22.86 \mathrm{e}$ & $22.91 \mathrm{e}$ \\
\hline compost + plastic sl & & & $29.49 a$ & $29.53 a$ & $29.36 \mathrm{a}$ & $29.43 a$ \\
\hline goat manure + plast & sheet & & $28.47 \mathrm{~b}$ & $28.46 \mathrm{~b}$ & $28.50 \mathrm{~b}$ & $28.70 \mathrm{~b}$ \\
\hline control $1+$ rice strav & & & $19.62 \mathrm{~h}$ & $19.80 \mathrm{~h}$ & $19.72 \mathrm{~h}$ & $19.83 \mathrm{~h}$ \\
\hline compost + rice stra & & & $25.68 \mathrm{c}$ & $25.94 \mathrm{c}$ & $25.62 \mathrm{c}$ & $25.61 \mathrm{c}$ \\
\hline goat manure + rice & aw & & $24.88 \mathrm{~d}$ & $24.68 d$ & $24.87 d$ & $25.09 \mathrm{~d}$ \\
\hline Effect of drip irrig & tion systems, & fertilization an & Iching & & & \\
\hline drip irrigation & Organic & & Number & / shoot & Shoo & (cm) \\
\hline systems & fertilization & soil mulching & 2012 & 2013 & 2012 & 2013 \\
\hline & control 1 & control 2 & $18.41 \mathrm{~m}$ & 18.611 & 19.081 & 19.171 \\
\hline & control 1 & plastic sheet & $22.57 \mathrm{~h}$ & $22.77 \mathrm{~g}$ & $22.52 \mathrm{~h}$ & $22.95 \mathrm{~g}$ \\
\hline & control 1 & rice straw & $22.33 \mathrm{~g}$ & $22.36 \mathrm{gh}$ & $22.14 \mathrm{~h}$ & $22.16 \mathrm{~h}$ \\
\hline & compost & control 2 & 23.57 & $23.70 \mathrm{f}$ & $23.75 \mathrm{~g}$ & $23.84 f$ \\
\hline sub surface & compost & plastic sheet & $30.19 a$ & $30.20 \mathrm{a}$ & $30.41 \mathrm{a}$ & $30.33 a$ \\
\hline & compost & rice straw & $29.09 \mathrm{~b}$ & $29.07 b$ & $29.18 b$ & $29.19 b$ \\
\hline & goat manure & control 2 & $20.04 \mathrm{k}$ & $20.07 j$ & $20.03 \mathrm{jk}$ & $20.12 \mathrm{jk}$ \\
\hline & goat manure & plastic sheet & $26.69 d$ & $26.82 \mathrm{~d}$ & $26.47 \mathrm{~d}$ & $26.22 d$ \\
\hline & goat manure & rice straw & $25.13 \mathrm{e}$ & $25.23 \mathrm{e}$ & $25.28 \mathrm{e}$ & $25.65 d$ \\
\hline
\end{tabular}


Improving growth and productivity of pomegranate fruit trees planted on sandy dunes slopes at...

\begin{tabular}{|l|l|l|c|c|c|c|}
\hline \multirow{5}{*}{ surface } & control 1 & control 2 & $16.52 \mathrm{n}$ & $16.55 \mathrm{~m}$ & $17.31 \mathrm{~m}$ & $17.53 \mathrm{~m}$ \\
\cline { 2 - 6 } & control 1 & plastic sheet & $21.23 \mathrm{j}$ & $21.42 \mathrm{i}$ & $21.18 \mathrm{i}$ & $21.31 \mathrm{i}$ \\
\cline { 2 - 6 } & control 1 & rice straw & $20.29 \mathrm{k}$ & $20.42 \mathrm{j}$ & $20.41 \mathrm{j}$ & $20.60 \mathrm{j}$ \\
\cline { 2 - 6 } & compost & control 2 & $21.92 \mathrm{i}$ & $21.98 \mathrm{~h}$ & $21.97 \mathrm{~h}$ & $21.99 \mathrm{~h}$ \\
\cline { 2 - 6 } & compost & plastic sheet & $28.79 \mathrm{~b}$ & $28.86 \mathrm{~b}$ & $28.31 \mathrm{c}$ & $28.53 \mathrm{c}$ \\
\cline { 2 - 6 } & compost & rice straw & $27.85 \mathrm{c}$ & $27.85 \mathrm{c}$ & $27.82 \mathrm{c}$ & $28.21 \mathrm{c}$ \\
\cline { 2 - 6 } & goat manure & control 2 & 19.211 & $25.52 \mathrm{k}$ & $19.41 \mathrm{kl}$ & $19.53 \mathrm{kl}$ \\
\cline { 2 - 7 } & goat manure & plastic sheet & $24.67 \mathrm{f}$ & $24.77 \mathrm{ef}$ & $25.00 \mathrm{e}$ \\
\cline { 2 - 6 } & goat manure & rice straw & $24.63 \mathrm{f}$ & $24.14 \mathrm{f}$ & $24.47 \mathrm{f}$ & $24.52 \mathrm{e}$ \\
\hline
\end{tabular}

Means having the same letter (s) in each column or interaction are not significantly different at 5\% level.

\section{Number of followers / shoot and fruit set \%}

Data in Table (5) showed that fruit set was significantly affected by all treatments in both seasons. It is obvious that subsurface drip irrigation system increased number of followers / shoot and fruit set $\%$ as compared with surface drip irrigation. In addition, compost gave the highest fruit set\% in both seasons. Furthermore, mulching the soil with plastic cover gave the best fruit set $\%$ as compared with control.

On the other hand, the interactions between irrigation systems and organic fertilization show that compost under sub surface drip irrigation gave the highest number of followers / shoot and fruit set \% in both seasons.

In addition, the interaction between irrigation systems and soil mulching show that number of followers / shoot and fruit set $\%$ increased with plastic cover under sub surface drip irrigation system as compared with surface drip irritation without mulching in both seasons.

However, the interactions between compost and mulching with plastic cover gave the highest number of followers / shoot and fruit set \% compared with control in both seasons.

Moreover the interactions between irrigation system, organic fertilization and soil mulching clear that compost and mulching the soil with plastic cover under sub surface drip irrigation gave the best number of followers / shoot (4.99 in first season and 5.00 in second seasons) and the highest fruit set (34.75 in first season and $35.00 \%$ in second seasons). In addition, there is no significant difference between compost and mulching the soil with plastic cover or rice straw under sub surface drip irrigation in fruit set at second seasons $134.74 \%$ in the second seasons). On the other hand, surface drip irrigation without fertilization and mulching gave the lowest fruit set \% in both seasons.

These results are in harmony with those obtained by Bryla, et al (2003) who found that sub surface drip irrigation improve vegetative growth of newly planted 'Crimson Lady' peach [Prunus persica (L.) Batsch] trees. Tiwari et al., (2014) recorded that plastic mulch and drip irrigation increase growth parameters in Sapota (Achras zapota) as compared with ring basin irrigation. Abd-Elaal et al., (2007) who founded that compost improving berry set in Superior grapevines in both seasons.

Table 5. Effect of irrigation systems, organic fertilization and soil mulching on number of followers / shoot and fruit set \% of Manfalouty cv. pomegranate trees during 2012 and 2013 seasons.

\begin{tabular}{|c|c|c|c|c|}
\hline \multirow{2}{*}{ Treatments } & \multicolumn{2}{|c|}{ Number of followers / shoot } & \multicolumn{2}{|c|}{ Fruit set \% } \\
\hline & 2012 & 2013 & 2012 & 2013 \\
\hline \multicolumn{5}{|c|}{ Effect of drip irrigation systems } \\
\hline sub surface & $3.10 \mathrm{a}$ & $3.23 \mathrm{a}$ & $28.42 \mathrm{a}$ & $28.73 \mathrm{a}$ \\
\hline surface & $2.68 b$ & $2.78 \mathrm{~b}$ & $26.74 b$ & $27.02 \mathrm{~b}$ \\
\hline \multicolumn{5}{|c|}{ Effect of organic fertilization } \\
\hline control 1 (without) & $2.09 \mathrm{c}$ & $2.14 \mathrm{c}$ & $23.85 \mathrm{c}$ & $24.09 \mathrm{c}$ \\
\hline compost & $3.74 a$ & $3.91 \mathrm{a}$ & $31.33 \mathrm{a}$ & $31.71 \mathrm{a}$ \\
\hline goat manure & $2.84 \mathrm{~b}$ & $2.95 \mathrm{~b}$ & $27.56 \mathrm{~b}$ & $27.83 \mathrm{~b}$ \\
\hline \multicolumn{5}{|l|}{ Effect of soil mulching } \\
\hline control 2 (without) & $2.05 \mathrm{c}$ & $2.12 \mathrm{c}$ & $23.42 \mathrm{c}$ & $23.64 \mathrm{c}$ \\
\hline plastic sheet & $3.45 \mathrm{a}$ & $3.55 \mathrm{a}$ & $29.99 \mathrm{a}$ & $30.30 \mathrm{a}$ \\
\hline rice straw & $3.17 \mathrm{~b}$ & $3.33 \mathrm{~b}$ & $29.33 b$ & $29.69 b$ \\
\hline \multicolumn{5}{|c|}{ Effect of drip irrigation systems and organic fertilization } \\
\hline sub surface + controll & $2.30 \mathrm{e}$ & $2.32 \mathrm{e}$ & $24.54 \mathrm{e}$ & $24.82 \mathrm{e}$ \\
\hline sub surface+ compost & $4.01 \mathrm{a}$ & $4.17 \mathrm{a}$ & $32.21 \mathrm{a}$ & $32.52 \mathrm{a}$ \\
\hline sub surface + goat manure & $3.00 \mathrm{c}$ & $3.18 \mathrm{c}$ & $28.51 \mathrm{c}$ & $28.83 \mathrm{c}$ \\
\hline surface + control 1 & $1.89 \mathrm{f}$ & $1.95 \mathrm{f}$ & $23.16 \mathrm{f}$ & $23.35 \mathrm{f}$ \\
\hline surface + compost & $3.48 \mathrm{~b}$ & $3.64 \mathrm{~b}$ & $30.44 b$ & $30.90 \mathrm{~b}$ \\
\hline surface + goat manure & $2.67 \mathrm{~d}$ & $2.72 \mathrm{~d}$ & $26.61 d$ & $26.83 d$ \\
\hline \multicolumn{5}{|c|}{ Effect of drip irrigation systems and soil mulching } \\
\hline sub surface + control 2 & $2.20 \mathrm{e}$ & $2.28 \mathrm{e}$ & $24.20 \mathrm{e}$ & $24.43 \mathrm{e}$ \\
\hline sub surface + plastic sheet & $3.77 \mathrm{a}$ & $3.84 \mathrm{a}$ & $30.76 \mathrm{a}$ & $31.16 \mathrm{a}$ \\
\hline sub surface + rice straw & $3.33 b$ & $3.56 \mathrm{~b}$ & $30.29 b$ & $30.59 b$ \\
\hline surface + control 2 & $1.89 \mathrm{f}$ & $1.95 \mathrm{f}$ & $22.64 f$ & $22.85 \mathrm{f}$ \\
\hline surface + plastic sheet & $3.13 \mathrm{c}$ & $3.26 \mathrm{c}$ & $29.20 \mathrm{c}$ & $29.43 c$ \\
\hline
\end{tabular}


Improving growth and productivity of pomegranate fruit trees planted on sandy dunes slopes at...

\begin{tabular}{|c|c|c|c|c|c|c|}
\hline \multicolumn{3}{|c|}{ surface + rice straw } & $3.01 \mathrm{~d}$ & $3.10 \mathrm{~d}$ & $28.37 \mathrm{~d}$ & $28.80 \mathrm{~d}$ \\
\hline \multicolumn{7}{|c|}{ Effect of organic fertilization and soil mulching } \\
\hline \multicolumn{3}{|c|}{ control $1+$ control 2} & $1.73 \mathrm{~h}$ & $1.74 \mathrm{i}$ & $20.73 \mathrm{i}$ & $20.91 \mathrm{i}$ \\
\hline \multicolumn{3}{|c|}{ compost + control 2} & $2.31 \mathrm{ef}$ & $2.39 \mathrm{f}$ & $25.62 \mathrm{f}$ & $25.92 \mathrm{f}$ \\
\hline \multicolumn{3}{|c|}{ goat manure + control 2} & $2.24 \mathrm{f}$ & $2.27 \mathrm{~g}$ & $25.21 \mathrm{~g}$ & $25.44 \mathrm{~g}$ \\
\hline \multicolumn{3}{|c|}{ control $1+$ plastic sheet } & $2.42 \mathrm{e}$ & $2.57 \mathrm{e}$ & $26.67 \mathrm{e}$ & $26.85 \mathrm{e}$ \\
\hline \multicolumn{3}{|c|}{ compost + plastic sheet } & $4.60 \mathrm{a}$ & $4.73 \mathrm{a}$ & $34.09 \mathrm{a}$ & $34.41 \mathrm{a}$ \\
\hline \multicolumn{3}{|c|}{ goat manure + plastic sheet } & $4.20 \mathrm{~b}$ & $4.42 b$ & $33.22 b$ & $33.87 \mathrm{~b}$ \\
\hline \multicolumn{3}{|c|}{ control $1+$ rice straw } & $1.99 \mathrm{~g}$ & $2.04 \mathrm{~h}$ & $22.86 \mathrm{~h}$ & $23.17 \mathrm{~h}$ \\
\hline \multicolumn{3}{|c|}{ compost + rice straw } & $3.44 \mathrm{c}$ & $3.52 \mathrm{c}$ & $30.25 \mathrm{c}$ & $30.56 \mathrm{c}$ \\
\hline \multicolumn{3}{|c|}{ goat manure + rice straw } & $3.07 \mathrm{~d}$ & $3.30 \mathrm{~d}$ & $29.56 \mathrm{~d}$ & $29.77 \mathrm{~d}$ \\
\hline \multicolumn{7}{|c|}{ Effect of drip irrigation systems, organic fertilization and soil mulching } \\
\hline \multirow{2}{*}{$\begin{array}{l}\text { drip irrigation } \\
\text { systems }\end{array}$} & \multirow{2}{*}{$\begin{array}{l}\text { organic } \\
\text { fertilization }\end{array}$} & \multirow{2}{*}{ soil mulching } & \multicolumn{2}{|c|}{ Number of followers / shoot } & \multicolumn{2}{|c|}{ Fruit set $\%$} \\
\hline & & & 2012 & 2013 & 2012 & 2013 \\
\hline \multirow{9}{*}{ sub surface } & control 1 & control 2 & $1.98 \mathrm{j}$ & $1.96 \mathrm{~m}$ & 21.460 & $21.70 \mathrm{o}$ \\
\hline & control 1 & plastic sheet & $2.51 \mathrm{fg}$ & $2.59 \mathrm{i}$ & $26.15 \mathrm{j}$ & $26.59 \mathrm{i}$ \\
\hline & control 1 & rice straw & $2.41 \mathrm{gh}$ & $2.42 \mathrm{j}$ & $26.00 \mathrm{j}$ & $26.18 \mathrm{j}$ \\
\hline & compost & control 2 & $2.63 \mathrm{f}$ & $2.80 \mathrm{~h}$ & $27.58 \mathrm{i}$ & $27.83 \mathrm{~h}$ \\
\hline & compost & plastic sheet & $4.99 \mathrm{a}$ & $5.00 \mathrm{a}$ & $34.75 \mathrm{a}$ & $35.00 \mathrm{a}$ \\
\hline & compost & rice straw & $4.39 \mathrm{~b}$ & $4.71 \mathrm{~b}$ & $34.31 \mathrm{~b}$ & $34.74 a$ \\
\hline & goat manure & control 2 & $2.00 \mathrm{ij}$ & $2.071 \mathrm{~m}$ & $23.56 \mathrm{~m}$ & $23.77 \mathrm{~m}$ \\
\hline & goat manure & plastic sheet & $3.80 \mathrm{c}$ & $3.92 \mathrm{e}$ & $31.38 \mathrm{e}$ & $31.89 \mathrm{~d}$ \\
\hline & goat manure & rice straw & $3.20 \mathrm{~d}$ & $3.55 \mathrm{f}$ & $30.57 f$ & $30.84 \mathrm{e}$ \\
\hline \multirow{9}{*}{ surface } & control 1 & control 2 & $1.49 \mathrm{k}$ & $1.53 n$ & $20.00 \mathrm{p}$ & $20.11 p$ \\
\hline & control 1 & plastic sheet & $2.11 \mathrm{ij}$ & $2.19 \mathrm{kl}$ & $25.08 \mathrm{k}$ & $25.25 \mathrm{k}$ \\
\hline & control 1 & rice straw & $2.07 \mathrm{ij}$ & $2.121 \mathrm{~m}$ & 24.421 & 24.691 \\
\hline & compost & control 2 & $2.21 \mathrm{hi}$ & $2.34 \mathrm{jk}$ & $25.76 \mathrm{j}$ & $25.88 \mathrm{j}$ \\
\hline & compost & plastic sheet & $4.22 \mathrm{~b}$ & $4.46 \mathrm{c}$ & $33.42 \mathrm{c}$ & $33.81 \mathrm{~b}$ \\
\hline & compost & rice straw & $4.01 \mathrm{c}$ & $4.13 d$ & $32.13 d$ & $33.00 \mathrm{c}$ \\
\hline & goat manure & control 2 & $1.99 \mathrm{ij}$ & $2.00 \mathrm{~m}$ & $22.15 n$ & $22.56 n$ \\
\hline & goat manure & plastic sheet & $3.07 \mathrm{de}$ & $3.12 \mathrm{~g}$ & $29.12 \mathrm{~g}$ & $29.22 \mathrm{f}$ \\
\hline & goat manure & rice straw & $2.95 \mathrm{e}$ & $3.05 \mathrm{~g}$ & $28.55 \mathrm{~h}$ & $28.70 \mathrm{~g}$ \\
\hline
\end{tabular}

Means having the same letter (s) in each column or interaction are not significantly different at $5 \%$ level.

\section{Fruit parameters (fruit physical characteristics)}

It could be noticed from tables (6 and 7) that all treatments are significantly increased fruit length, diameter, weight and grains weight than the control in both seasons. Subsurface drip irrigation system increased fruit length, diameter, weight and grains weight in both seasons as compared with surface drip irrigation. In addition, compost gave the highest fruit length, diameter, weight and grains weight in both seasons. Furthermore, mulching the soil with plastic cover gave the best fruit length, diameter, weight and grains weight as compared with control.

On the other hand, the interactions between compost and sub surface drip irrigation gave the highest fruit length $\left(8.20\right.$ in the $1^{\text {st }}$ and $8.26 \mathrm{~cm}$ in the $2^{\text {nd }}$ season), fruit diameter $(9.30$ in first season and $9.58 \mathrm{~cm}$ in second seasons respectively), fruit weight (404.21 in the $1^{\text {st }}$ and $405.03 \mathrm{~g}$ in the $2^{\text {nd }}$ season) and grains weight (315.52 in first season and $317.37 \mathrm{~g}$ in second seasons respectively).

Moreover, the interaction between plastic cover and sub surface drip irrigation was the best effect in fruit length, diameter, weight and grains weight than surface drip irritation without mulching in both seasons.

In addition, the interactions between compost and mulching with plastic cover gave the highest fruit length, diameter, weight and grains weight as compared with control in both seasons.

Moreover the interactions between compost and mulching the soil with plastic cover under sub surface drip irrigation gave the best fruit length, diameter, weight and grains weight in both seasons. On the other hand, surface drip irrigation without fertilization and mulching gave the less fruit length, diameter, weight and grains weight in both seasons.

These results are in parallel with those obtained by Bryla, et al., (2003) who founded that sub surface drip irrigation improve vegetative growth of newly planted 'Crimson Lady' peach [Prunus persica (L.) Batsch] trees. In addition, (Abd-ELaal et al., 2007) recorded that the compost increasing cluster weight, berry weight and dimensions on superior grapevines. 
Improving growth and productivity of pomegranate fruit trees planted on sandy dunes slopes at...

Table 6. Effect of irrigation systems, organic fertilization and soil mulching on fruit length and fruit diameter of Manfalouty cv. pomegranate trees during 2012 and 2013 seasons.

\begin{tabular}{|c|c|c|c|c|c|c|}
\hline \multirow{2}{*}{\multicolumn{3}{|c|}{ Treatments }} & \multicolumn{2}{|c|}{ Fruit length $(\mathrm{cm})$} & \multicolumn{2}{|c|}{ Fruit diameter $(\mathrm{cm})$} \\
\hline & & & 2012 & 2013 & 2012 & 2013 \\
\hline \multicolumn{7}{|c|}{ Effect of drip irrigation systems } \\
\hline \multicolumn{3}{|c|}{ sub surface } & $7.88 \mathrm{a}$ & $7.93 \mathrm{a}$ & $8.77 \mathrm{a}$ & $8.91 \mathrm{a}$ \\
\hline \multicolumn{3}{|l|}{ surface } & $7.71 \mathrm{~b}$ & $7.76 \mathrm{~b}$ & $8.55 \mathrm{~b}$ & $8.57 \mathrm{~b}$ \\
\hline \multicolumn{7}{|c|}{ Effect of organic fertilization } \\
\hline \multicolumn{3}{|c|}{ control 1 (without ) } & $7.50 \mathrm{c}$ & $7.54 \mathrm{c}$ & $8.14 \mathrm{c}$ & $8.17 \mathrm{c}$ \\
\hline \multicolumn{3}{|l|}{ compost } & $8.10 \mathrm{a}$ & $8.16 \mathrm{a}$ & $9.21 \mathrm{a}$ & $9.38 \mathrm{a}$ \\
\hline \multicolumn{3}{|l|}{ goat manure } & $7.80 \mathrm{~b}$ & $7.84 \mathrm{~b}$ & $8.63 \mathrm{~b}$ & $8.69 \mathrm{~b}$ \\
\hline \multicolumn{7}{|c|}{ Effect of soil mulching } \\
\hline \multicolumn{3}{|c|}{ control 2 (without) } & $7.46 \mathrm{c}$ & $7.49 \mathrm{c}$ & $7.97 \mathrm{c}$ & $8.02 \mathrm{c}$ \\
\hline \multicolumn{3}{|l|}{ plastic sheet } & $8.01 \mathrm{a}$ & $8.05 \mathrm{a}$ & $9.08 \mathrm{a}$ & $9.16 \mathrm{a}$ \\
\hline \multicolumn{3}{|c|}{ rice straw } & $7.93 \mathrm{~b}$ & $7.99 \mathrm{~b}$ & $8.93 \mathrm{~b}$ & $9.05 \mathrm{~b}$ \\
\hline \multicolumn{7}{|c|}{ Effect of drip irrigation systems and organic fertilization } \\
\hline \multicolumn{3}{|c|}{ sub surface + controll } & $7.64 \mathrm{e}$ & $7.63 \mathrm{e}$ & $8.29 \mathrm{e}$ & $8.35 \mathrm{e}$ \\
\hline \multicolumn{3}{|c|}{ sub surface+ compost } & $8.20 \mathrm{a}$ & $8.26 \mathrm{a}$ & $9.30 \mathrm{a}$ & $9.58 \mathrm{a}$ \\
\hline sub surface + goat $\mathrm{m}$ & ure & & $7.82 \mathrm{c}$ & $7.88 \mathrm{c}$ & $8.70 \mathrm{c}$ & $8.80 \mathrm{c}$ \\
\hline surface + control1 & & & $7.37 \mathrm{f}$ & $7.44 \mathrm{f}$ & $7.99 \mathrm{f}$ & $7.98 \mathrm{f}$ \\
\hline surface + compost & & & $8.01 \mathrm{~b}$ & $8.06 \mathrm{~b}$ & $9.11 \mathrm{~b}$ & $9.18 \mathrm{~b}$ \\
\hline surface + goat manur & & & $7.78 \mathrm{~d}$ & $7.79 \mathrm{~d}$ & $8.55 \mathrm{~d}$ & $8.56 \mathrm{~d}$ \\
\hline Effect of drip irrig & on systems ar & lulching & & & & \\
\hline sub surface + contro & & & $7.55 \mathrm{e}$ & $7.58 \mathrm{e}$ & $8.07 \mathrm{e}$ & 8.15[ \\
\hline sub surface + plastic & eet & & $8.10 \mathrm{a}$ & $8.14 \mathrm{a}$ & $9.17 \mathrm{a}$ & $9.32 \mathrm{a}$ \\
\hline sub surface + rice str & & & $8.00 \mathrm{~b}$ & $8.05 \mathrm{~b}$ & $9.06 \mathrm{~b}$ & $9.27 \mathrm{~b}$ \\
\hline surface + control2 & & & $7.36 \mathrm{f}$ & $7.40 \mathrm{f}$ & $7.87 \mathrm{f}$ & $7.90 \mathrm{f}$ \\
\hline surface + plastic shee & & & $7.93 \mathrm{c}$ & $7.97 \mathrm{c}$ & $8.98 \mathrm{c}$ & $9.00 \mathrm{c}$ \\
\hline surface + rice straw & & & $7.86 \mathrm{~d}$ & $7.92 \mathrm{~d}$ & $8.81 \mathrm{~d}$ & $8.82 \mathrm{~d}$ \\
\hline Effect of organic fe & ilization and & ching & & & & \\
\hline control $1+$ control & & & $7.15 \mathrm{~h}$ & $7.21 \mathrm{~g}$ & $7.27 \mathrm{i}$ & $7.33 \mathrm{i}$ \\
\hline compost + control 2 & & & $7.73 \mathrm{e}$ & $7.73 \mathrm{e}$ & $8.64 \mathrm{f}$ & $8.65 \mathrm{f}$ \\
\hline goat manure + cont & & & $7.64 \mathrm{f}$ & $7.67 \mathrm{e}$ & $8.51 \mathrm{~g}$ & $8.52 \mathrm{~g}$ \\
\hline control $1+$ plastic $s$ & & & $7.79 \mathrm{~d}$ & $7.80 \mathrm{~d}$ & $8.70 \mathrm{e}$ & $8.76 \mathrm{e}$ \\
\hline compost + plastic sh & & & $8.32 \mathrm{a}$ & $8.40 \mathrm{a}$ & $9.53 \mathrm{a}$ & $9.74 \mathrm{a}$ \\
\hline goat manure + plast & sheet & & $8.20 \mathrm{~b}$ & $8.28 \mathrm{~b}$ & $9.38 \mathrm{~b}$ & $9.64 b$ \\
\hline control $1+$ rice straw & & & $7.43 \mathrm{~g}$ & $7.47 \mathrm{f}$ & $7.94 \mathrm{~h}$ & $7.97 \mathrm{~h}$ \\
\hline compost + rice stray & & & $7.99 \mathrm{c}$ & $8.04 \mathrm{c}$ & $9.04 \mathrm{c}$ & $9.08 \mathrm{c}$ \\
\hline goat manure + rice $s$ & & & $7.97 \mathrm{c}$ & $8.00 \mathrm{c}$ & $8.91 \mathrm{~d}$ & $8.98 \mathrm{~d}$ \\
\hline Effect of drip irrig & ion systems, & ertilization anc & Iching & & & \\
\hline drip irrigation & organic & & & & & ter \\
\hline systems & fertilization & Soll muicning & 2012 & 2013 & 2012 & 2013 \\
\hline & control 1 & control 2 & $7.30 \mathrm{~m}$ & $7.31 \mathrm{~m}$ & $7.42 \mathrm{p}$ & $7.51 \mathrm{p}$ \\
\hline & control 1 & plastic sheet & $7.85 \mathrm{~g}$ & $7.83 \mathrm{~g}$ & $8.75 \mathrm{i}$ & $8.80 \mathrm{i}$ \\
\hline & control 1 & rice straw & $7.76 \mathrm{~h}$ & $7.76 \mathrm{gh}$ & $8.72 \mathrm{j}$ & $8.75 \mathrm{j}$ \\
\hline & compost & control 2 & $7.89 \mathrm{~g}$ & $7.91 \mathrm{f}$ & $8.80 \mathrm{~h}$ & $8.82 \mathrm{~h}$ \\
\hline sub surface & compost & plastic sheet & $8.43 a$ & $8.50 \mathrm{a}$ & $9.65 a$ & $9.97 \mathrm{a}$ \\
\hline & compost & rice straw & $8.27 \mathrm{~b}$ & $8.37 \mathrm{~b}$ & $9.45 \mathrm{~b}$ & $9.96 \mathrm{a}$ \\
\hline & goat manure & control 2 & 7.451 & $7.51 \mathrm{k}$ & $8.00 \mathrm{~m}$ & $8.10 \mathrm{n}$ \\
\hline & goat manure & plastic sheet & $8.01 \mathrm{e}$ & $8.10 \mathrm{~d}$ & $9.11 \mathrm{e}$ & $9.18 \mathrm{~d}$ \\
\hline & goat manure & rice straw & 7.99ef & $8.03 \mathrm{de}$ & $9.00 \mathrm{f}$ & $9.11 \mathrm{e}$ \\
\hline & control 1 & control 2 & $7.00 \mathrm{n}$ & $7.10 \mathrm{n}$ & $7.13 q$ & $7.14 \mathrm{q}$ \\
\hline & control 1 & plastic sheet & $7.60 \mathrm{j}$ & $7.62 \mathrm{ij}$ & 8.531 & 8.501 \\
\hline & control 1 & rice straw & $7.51 \mathrm{k}$ & $7.59 \mathrm{jk}$ & $8.30 \mathrm{~m}$ & $8.29 \mathrm{~m}$ \\
\hline & compost & control 2 & $7.69 \mathrm{i}$ & 7.69hi & $8.61 \mathrm{k}$ & $8.70 \mathrm{k}$ \\
\hline surface & compost & plastic sheet & $8.20 \mathrm{c}$ & $8.30 \mathrm{~b}$ & $9.42 \mathrm{c}$ & $9.51 \mathrm{~b}$ \\
\hline & compost & rice straw & $8.13 \mathrm{~d}$ & $8.20 \mathrm{c}$ & $9.32 \mathrm{~d}$ & $9.31 \mathrm{c}$ \\
\hline & goat manure & control 2 & 7.411 & 7.421 & $7.87 \mathrm{o}$ & 7.840 \\
\hline & goat manure & plastic sheet & 7.98ef & 7.98ef & $8.98 \mathrm{~g}$ & $8.98 \mathrm{f}$ \\
\hline & goat manure & rice straw & $7.94 \mathrm{f}$ & 7.96ef & $8.81 \mathrm{~h}$ & $8.85 \mathrm{~g}$ \\
\hline
\end{tabular}

Means having the same letter (s) in each column or interaction are not significantly different at $5 \%$ level.

Table 7. Effect of irrigation systems, organic fertilization and soil mulching on fruit weight and grains weight of Manfalouty cv. pomegranate trees during 2012 and 2013 seasons.

\begin{tabular}{|l|c|c|c|c|}
\hline \multirow{2}{*}{ Treatments } & \multicolumn{2}{|c|}{ Fruit weight(g) } & \multicolumn{3}{c|}{ Grains weight (g) } \\
\cline { 2 - 5 } & $\mathbf{2 0 1 2}$ & $\mathbf{2 0 1 3}$ & $\mathbf{2 0 1 3}$ \\
\hline Effect of drip irrigation systems & $385.95 \mathrm{a}$ & $388.22 \mathrm{a}$ & $287.88 \mathrm{a}$ & $290.33 \mathrm{a}$ \\
\hline sub surface & $377.17 \mathrm{~b}$ & $379.83 \mathrm{~b}$ & $268.97 \mathrm{~b}$ & $274.70 \mathrm{~b}$ \\
\hline surface & \multicolumn{5}{|l}{$93 \mid \mathrm{Page}$} \\
\hline
\end{tabular}


Improving growth and productivity of pomegranate fruit trees planted on sandy dunes slopes at...

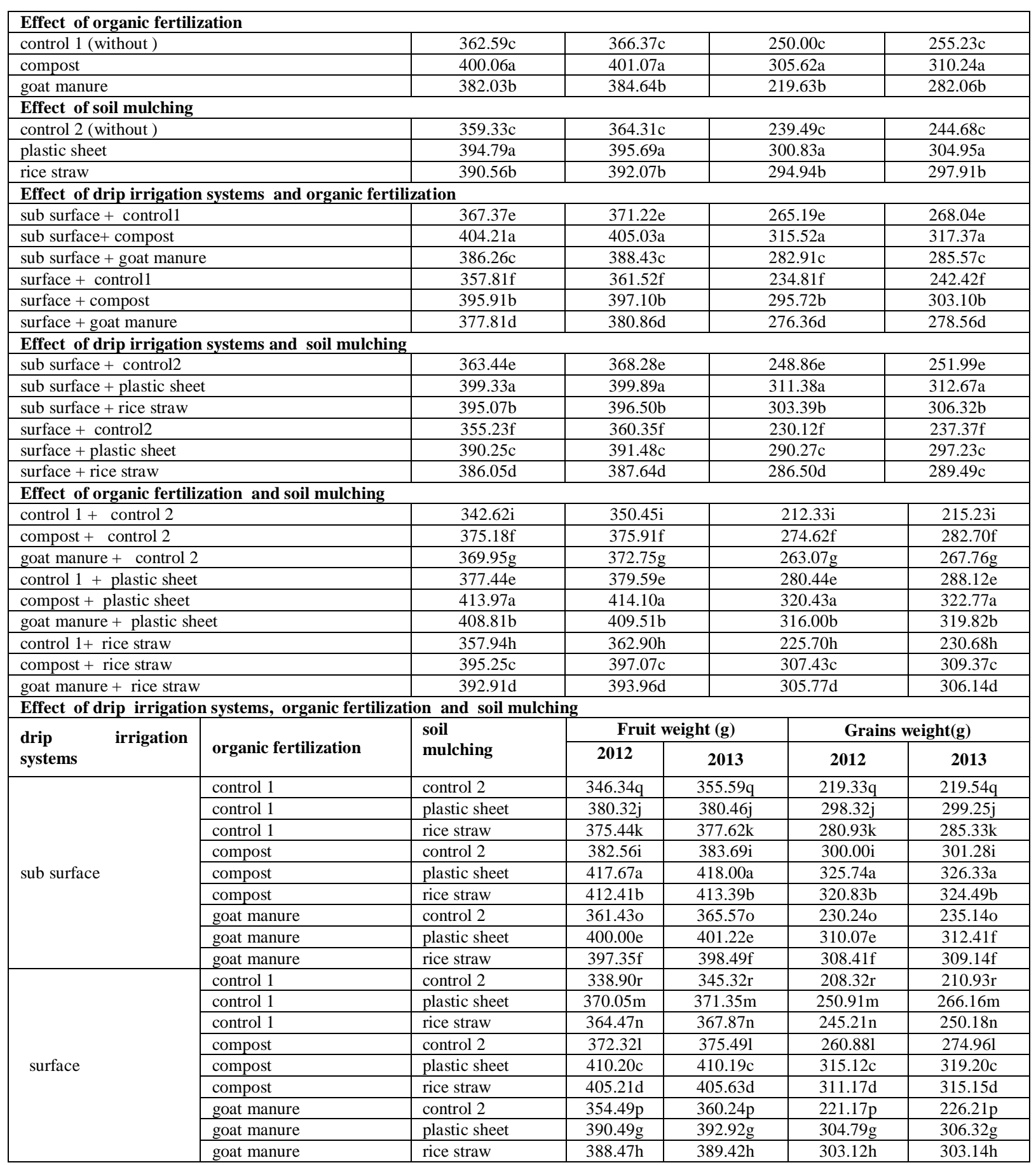

Means having the same letter (s) in each column or interaction are not significantly different at $5 \%$ level.

\section{Fruit quality (fruit chemical characteristics)}

It could be noticed from (8 and 9) that all treatments significantly increased TSS\%, total sugar content and vitamin $\mathrm{C} \mathrm{mg} / 100 \mathrm{~g}$ than the control in both seasons. Sub surface drip irrigation system increased TSS, total sugar, vitamin $\mathrm{C}$ and decrease total acidity in both seasons as compared with surface drip irrigation. In addition, compost gave the highest TSS, total sugar content, vitamin C in both seasons. Furthermore, mulching the soil with plastic cover gave the best fruit TSS, total sugar content, vitamin C and the lowest total acidity.

On the other hand, the interactions between compost and sub surface drip irrigation gave the highest TSS (17.15 in the $1^{\text {st }}$ and $17.23 \%$ in the $2^{\text {nd }}$ season), total sugar content $(13.94$ in first season and $14.07 \%$ in second seasons respectively), vitamin C $(16.61 \mathrm{mg} / 100 \mathrm{~g}$ in both seasons) and the lowest total acidity (1.12 in the $1^{\text {st }}$ and $1.09 \%$ in the $2^{\text {nd }}$ season).

While, the interaction between plastic cover and sub surface drip irrigation was the best effect in TSS, total sugar content, total acidity and vitamin $\mathrm{C}$ than surface drip irritation without mulching in both seasons. 
Improving growth and productivity of pomegranate fruit trees planted on sandy dunes slopes at...

In addition, the interactions between compost and mulching with plastic cover gave the highest TSS, total sugar content, vitamin $\mathrm{C}$ and the lowest total acidity in both seasons.

Moreover the interactions between compost and mulching the soil with plastic cover or rice straw under sub surface drip irrigation gave the best TSS and total sugar content in the first season, but in the second season, plastic cover under sub surface drip irrigation with compost fertilization gave the best TSS and total sugar content. In addition, there was non significant deference between plastic cover and rise straw under sub surface drip irrigation with compost fertilization in vitamin $\mathrm{C}$ in both seasons. Furthermore, compost and mulching the soil with rice straw under sub surface drip irrigation gave the lowest total acidity in first season but there was non significant deference between rice straw and plastic cover under sub surface drip irrigation with compost fertilization in total acidity. On the other hand, surface drip irrigation without fertilization and mulching gave less TSS, total sugar content, vitamin $\mathrm{C}$ and increase total acidity in both seasons.

These results are in harmony with those obtained by Bryla, et al., (2003) founded that sub surface drip irrigation improve vegetative growth of newly planted 'Crimson Lady' peach [Prunus persica (L.) Batsch] trees. In addition, (Abd-ELaal et al., 2007) recorded that the compost increases percentage of total soluble solids while decreasing the total acidity of the juice on superior grapevines.

Table 8. Effect of irrigation systems, organic fertilization and soil mulching on TSS\% and total sugar content of Manfalouty cv. pomegranate trees during 2012 and 2013 seasons.

\begin{tabular}{|c|c|c|c|c|c|c|}
\hline \multirow{2}{*}{\multicolumn{3}{|c|}{ Treatments }} & \multicolumn{2}{|c|}{ TSS\% } & \multicolumn{2}{|c|}{ Total sugar \% } \\
\hline & & & 2012 & 2013 & 2012 & 2013 \\
\hline \multicolumn{7}{|c|}{ Effect of drip irrigation systems } \\
\hline \multicolumn{3}{|c|}{ sub surface } & $16.53 \mathrm{a}$ & $16.61 \mathrm{a}$ & $13.27 \mathrm{a}$ & $13.38 \mathrm{a}$ \\
\hline \multicolumn{3}{|l|}{ surface } & $16.23 \mathrm{~b}$ & $16.33 \mathrm{~b}$ & $12.97 \mathrm{~b}$ & $13.04 \mathrm{~b}$ \\
\hline \multicolumn{7}{|c|}{ Effect of organic fertilization } \\
\hline \multicolumn{3}{|c|}{ control 1 (without) } & $15.76 \mathrm{c}$ & $15.86 \mathrm{c}$ & $12.97 \mathrm{c}$ & $12.48 \mathrm{c}$ \\
\hline \multicolumn{3}{|l|}{ compost } & $16.99 \mathrm{a}$ & $17.03 \mathrm{a}$ & $13.80 \mathrm{a}$ & $13.91 \mathrm{a}$ \\
\hline \multicolumn{3}{|l|}{ goat manure } & $16.40 \mathrm{~b}$ & $16.51 \mathrm{~b}$ & $13.15 b$ & $13.24 \mathrm{~b}$ \\
\hline \multicolumn{7}{|c|}{ Effect of soil mulching } \\
\hline \multicolumn{3}{|c|}{ control 2 (without) } & $15.67 \mathrm{c}$ & $15.81 \mathrm{c}$ & $12.41 \mathrm{c}$ & $12.50 \mathrm{c}$ \\
\hline \multicolumn{3}{|l|}{ plastic sheet } & $16.80 \mathrm{a}$ & $16.85 \mathrm{a}$ & $13.55 \mathrm{a}$ & $13.67 \mathrm{a}$ \\
\hline \multicolumn{3}{|c|}{ rice straw } & $16.68 \mathrm{~b}$ & $16.74 \mathrm{~b}$ & $13.39 b$ & $13.50 \mathrm{~b}$ \\
\hline \multicolumn{7}{|c|}{ Effect of drip irrigation systems and organic fertilization } \\
\hline \multicolumn{3}{|c|}{ sub surface + control1 } & $15.96 \mathrm{e}$ & $16.03 \mathrm{e}$ & $12.55 \mathrm{e}$ & $12.66 \mathrm{e}$ \\
\hline \multicolumn{3}{|l|}{ sub surface+ compost } & $17.15 \mathrm{a}$ & $17.23 \mathrm{a}$ & $13.94 \mathrm{a}$ & $14.07 \mathrm{a}$ \\
\hline \multicolumn{3}{|c|}{ sub surface + goat manure } & $16.49 \mathrm{c}$ & $16.57 \mathrm{c}$ & $13.30 \mathrm{c}$ & $13.40 \mathrm{cb}$ \\
\hline \multicolumn{3}{|c|}{ surface + control1 } & $15.55 \mathrm{f}$ & $15.70 \mathrm{f}$ & $12.27 \mathrm{f}$ & $12.30 \mathrm{f}$ \\
\hline \multicolumn{3}{|l|}{ surface + compost } & $16.83 \mathrm{~b}$ & $16.64 \mathrm{~b}$ & $13.65 \mathrm{~b}$ & $13.75 \mathrm{c}$ \\
\hline \multicolumn{3}{|c|}{ surface + goat manure } & $16.31 \mathrm{~d}$ & $16.45 \mathrm{~d}$ & $13.00 \mathrm{~d}$ & $13.08 \mathrm{~d}$ \\
\hline Effect of drip irrig & ion systems al & nulching & & & & \\
\hline sub surface + control & & & $15.85 \mathrm{e}$ & $15.96 \mathrm{e}$ & $12.57 \mathrm{e}$ & $12.63 \mathrm{e}$ \\
\hline sub surface + plastic & leet & & $16.92 \mathrm{a}$ & $17.00 \mathrm{a}$ & $13.72 \mathrm{a}$ & $13.88 \mathrm{a}$ \\
\hline sub surface + rice str & & & $16.83 \mathrm{~b}$ & $16.86 \mathrm{~b}$ & $13.51 \mathrm{~b}$ & $13.63 b$ \\
\hline surface + control 2 & & & $15.50 \mathrm{f}$ & $15.65 \mathrm{f}$ & $12.26 \mathrm{f}$ & $12.31 \mathrm{f}$ \\
\hline surface + plastic shee & & & $16.68 \mathrm{c}$ & $16.70 \mathrm{c}$ & $13.39 \mathrm{c}$ & $13.46 \mathrm{c}$ \\
\hline surface + rice straw & & & $16.52 \mathrm{~d}$ & $16.62 d$ & $13.27 \mathrm{~d}$ & $13.36 \mathrm{~d}$ \\
\hline Effect of organic fe & ilization and & hing & & & & \\
\hline control $1+$ control 2 & & & $15.07 \mathrm{i}$ & $15.21 \mathrm{i}$ & $12.13 \mathrm{~h}$ & $12.16 \mathrm{i}$ \\
\hline compost + control 2 & & & $16.16 \mathrm{f}$ & $16.25 \mathrm{f}$ & $12.58 \mathrm{f}$ & $12.70 \mathrm{f}$ \\
\hline goat manure + contr & & & $16.05 \mathrm{~g}$ & $16.13 \mathrm{~g}$ & $12.53 \mathrm{f}$ & $12.58 \mathrm{~g}$ \\
\hline control $1+$ plastic s & & & $16.28 \mathrm{e}$ & $16.40 \mathrm{e}$ & $12.78 \mathrm{e}$ & $12.89 \mathrm{e}$ \\
\hline compost + plastic sh & & & $17.41 \mathrm{a}$ & $17.41 \mathrm{a}$ & $14.36 \mathrm{a}$ & $14.48 \mathrm{a}$ \\
\hline goat manure + plasti & sheet & & $17.28 \mathrm{~b}$ & $17.29 \mathrm{~b}$ & $14.25 \mathrm{~b}$ & $14.37 \mathrm{~b}$ \\
\hline control $1+$ rice straw & & & $15.68 \mathrm{~h}$ & $15.81 \mathrm{~h}$ & $12.33 \mathrm{~g}$ & $1236 \mathrm{~h}$ \\
\hline compost + rice straw & & & $16.83 \mathrm{c}$ & $16.90 \mathrm{c}$ & $13.73 \mathrm{c}$ & $13.83 \mathrm{c}$ \\
\hline goat manure + rice $s$ & & & $16.71 \mathrm{~d}$ & $16.82 \mathrm{~d}$ & $13.40 \mathrm{~d}$ & $13.53 \mathrm{~d}$ \\
\hline Effect of drip irrig & tion systems, & fertilization an & ching & & & \\
\hline drip irrigation & organic & & & & Tota & $\%$ \\
\hline systems & fertilization & Soll mulchıng & 2012 & 2013 & 2012 & 2013 \\
\hline & control 1 & control 2 & $15.35 \mathrm{~m}$ & $15.36 \mathrm{p}$ & $12.25 \mathrm{j}$ & $12.27 p$ \\
\hline & control 1 & plastic sheet & $16.33 \mathrm{fg}$ & $16.46 \mathrm{i}$ & $12.74 \mathrm{~g}$ & $12.98 \mathrm{j}$ \\
\hline & control 1 & rice straw & $16.21 \mathrm{gh}$ & $16.28 \mathrm{j}$ & $12.66 \mathrm{~g}$ & $12.74 \mathrm{k}$ \\
\hline & compost & control 2 & $16.45 \mathrm{ef}$ & $16.66 \mathrm{~h}$ & $13.10 \mathrm{f}$ & $12.23 \mathrm{i}$ \\
\hline sub surface & compost & plastic sheet & $17.56 \mathrm{a}$ & $17.56 \mathrm{a}$ & $14.41 \mathrm{a}$ & $14.55 \mathrm{a}$ \\
\hline & compost & rice straw & $17.44 \mathrm{a}$ & $17.46 \mathrm{~b}$ & $14.32 \mathrm{a}$ & $14.43 b$ \\
\hline & goat manure & control 2 & $15.75 \mathrm{k}$ & $15.86 \mathrm{n}$ & 12.35hij & $12.37 \mathrm{~m}$ \\
\hline & goat manure & plastic sheet & $16.87 \mathrm{~d}$ & $16.99 \mathrm{e}$ & $14.00 \mathrm{c}$ & $14.11 \mathrm{e}$ \\
\hline & goat manure & rice straw & $16.85 \mathrm{~d}$ & $16.86 \mathrm{f}$ & $13.55 \mathrm{~d}$ & $13.71 \mathrm{f}$ \\
\hline
\end{tabular}


Improving growth and productivity of pomegranate fruit trees planted on sandy dunes slopes at...

\begin{tabular}{|l|l|l|l|l|l|l|}
\hline \multirow{5}{*}{ surface } & control 2 & $14.78 \mathrm{n}$ & $15.07 \mathrm{q}$ & $12.00 \mathrm{k}$ & $12.05 \mathrm{q}$ \\
\cline { 2 - 7 } & control 1 & plastic sheet & $16.00 \mathrm{ij}$ & $16.05 \mathrm{l}$ & $12.42 \mathrm{hi}$ & $12.42 \mathrm{~m}$ \\
\cline { 2 - 7 } & control 1 & rice straw & $15.88 \mathrm{jk}$ & $15.98 \mathrm{~m}$ & $12.41 \mathrm{hi}$ & $12.42 \mathrm{~m}$ \\
\cline { 2 - 7 } & compost & control 2 & $16.11 \mathrm{hi}$ & $16.13 \mathrm{k}$ & $12.47 \mathrm{~h}$ & 12.541 \\
\cline { 2 - 7 } & compost & plastic sheet & $17.26 \mathrm{~b}$ & $17.26 \mathrm{c}$ & $14.30 \mathrm{a}$ & $14.40 \mathrm{c}$ \\
\cline { 2 - 7 } & compost & rice straw & $17.12 \mathrm{c}$ & $17.12 \mathrm{~d}$ & $14.18 \mathrm{~b}$ & $14.31 \mathrm{~d}$ \\
\cline { 2 - 7 } & goat manure & control 2 & 15.601 & $15.76 \mathrm{o}$ & $12.31 \mathrm{ij}$ & $12.35 \mathrm{o}$ \\
\cline { 2 - 7 } & goat manure & plastic sheet & $16.78 \mathrm{~d}$ & $16.80 \mathrm{~g}$ & $13.45 \mathrm{~d}$ & $13.55 \mathrm{~g}$ \\
\cline { 2 - 7 } & goat manure & rice straw & $16.56 \mathrm{e}$ & $16.77 \mathrm{~g}$ & $13.24 \mathrm{e}$ & $13.35 \mathrm{~h}$ \\
\hline
\end{tabular}

Means having the same letter (s) in each column or interaction are not significantly different at $5 \%$ level.

Table 9. Effect of irrigation systems, organic fertilization and soil mulching on total acidity and vitamin $\mathrm{C}$ of Manfalouty cv. pomegranate trees during 2012 and 2013 seasons.

\begin{tabular}{|c|c|c|c|c|c|c|}
\hline \multirow{2}{*}{\multicolumn{3}{|c|}{ Treatments }} & \multicolumn{2}{|c|}{ Total acidity \% } & \multicolumn{2}{|c|}{ Vitamin C mg/100g } \\
\hline & & & 2012 & 2013 & 2012 & 2013 \\
\hline \multicolumn{7}{|c|}{ Effect of drip irrigation systems } \\
\hline \multicolumn{3}{|c|}{ sub surface } & $1.24 \mathrm{~b}$ & $1.22 \mathrm{~b}$ & $15.97 \mathrm{a}$ & $16.02 \mathrm{a}$ \\
\hline \multicolumn{3}{|c|}{ surface } & $1.29 \mathrm{a}$ & $1.29 \mathrm{a}$ & $15.53 \mathrm{~b}$ & $15.63 \mathrm{~b}$ \\
\hline \multicolumn{7}{|c|}{ Effect of organic fertilization } \\
\hline \multicolumn{3}{|c|}{ Control 1(without) } & $1.38 \mathrm{a}$ & $1.39 \mathrm{a}$ & $14.94 \mathrm{c}$ & $15.04 \mathrm{c}$ \\
\hline \multicolumn{3}{|l|}{ compost } & $1.15 \mathrm{c}$ & $1.12 \mathrm{c}$ & $16.44 \mathrm{a}$ & $16.48 \mathrm{a}$ \\
\hline \multicolumn{3}{|l|}{ goat manure } & $1.26 \mathrm{~b}$ & $1.25 \mathrm{~b}$ & $15.87 \mathrm{~b}$ & $15.97 \mathrm{~b}$ \\
\hline \multicolumn{7}{|c|}{ Effect of soil mulching } \\
\hline \multicolumn{3}{|c|}{ control 2 (without) } & $1.39 \mathrm{a}$ & $1.40 \mathrm{a}$ & $14.69 \mathrm{c}$ & $14.84 \mathrm{c}$ \\
\hline \multicolumn{3}{|l|}{ plastic sheet } & $1.20 \mathrm{c}$ & $1.17 \mathrm{c}$ & $16.31 \mathrm{a}$ & $16.35 \mathrm{a}$ \\
\hline \multicolumn{3}{|c|}{ rice straw } & $1.21 \mathrm{~b}$ & $1.20 \mathrm{~b}$ & $16.24 \mathrm{~b}$ & $16.30 \mathrm{~b}$ \\
\hline \multicolumn{7}{|c|}{ Effect of drip irrigation systems and organic fertilization } \\
\hline \multicolumn{3}{|c|}{ sub surface + control 1} & $1.34 \mathrm{~b}$ & $1.36 \mathrm{~b}$ & $15.31 \mathrm{e}$ & $15.40 \mathrm{e}$ \\
\hline \multicolumn{3}{|l|}{ sub surface+ compost } & $1.12 \mathrm{f}$ & $1.09 \mathrm{f}$ & $16.61 \mathrm{a}$ & $16.61 \mathrm{a}$ \\
\hline sub surface + goat mi & ure & & $1.25 \mathrm{~d}$ & $1.23 \mathrm{~d}$ & $15.98 \mathrm{c}$ & $16.06 \mathrm{c}$ \\
\hline surface + control 1 & & & $1.41 \mathrm{a}$ & $1.43 \mathrm{a}$ & $14.57 \mathrm{f}$ & $14.68 \mathrm{f}$ \\
\hline surface + compost & & & $1.18 \mathrm{e}$ & $1.16 \mathrm{e}$ & $16.26 \mathrm{~b}$ & $16.35 b$ \\
\hline surface + goat manur & & & $1.28 \mathrm{c}$ & $1.28 \mathrm{c}$ & $15.76 \mathrm{~d}$ & $15.87 \mathrm{~d}$ \\
\hline Effect of drip irriga & on systems an & ulching & & & & \\
\hline sub surface + control & & & $1.36 \mathrm{~b}$ & $1.37 \mathrm{~b}$ & $14.90 \mathrm{~d}$ & $15.00 \mathrm{~d}$ \\
\hline sub surface + plastic & eet & & $1.18 \mathrm{e}$ & $1.13 \mathrm{f}$ & $16.53 \mathrm{a}$ & $16.53 \mathrm{a}$ \\
\hline sub surface + rice str & & & $1.17 \mathrm{e}$ & $1.17 \mathrm{e}$ & $16.46 \mathrm{a}$ & $16.53 \mathrm{a}$ \\
\hline surface + control 2 & & & $1.41 \mathrm{a}$ & $1.43 \mathrm{a}$ & $14.48 \mathrm{e}$ & $14.67 \mathrm{e}$ \\
\hline surface + plastic shee & & & $1.21 \mathrm{~d}$ & $1.22 \mathrm{~d}$ & $16.10 \mathrm{~b}$ & $16.17 \mathrm{~b}$ \\
\hline surface + rice straw & & & $1.24 \mathrm{c}$ & $1.23 \mathrm{c}$ & $16.02 \mathrm{c}$ & $16.06 \mathrm{c}$ \\
\hline Effect of organic fe & lization and & hing & & & & \\
\hline control $1+$ control 2 & & & $1.53 \mathrm{a}$ & $1.54 \mathrm{a}$ & $14.04 \mathrm{f}$ & $14.16 \mathrm{~g}$ \\
\hline compost + control 2 & & & $1.29 \mathrm{~d}$ & $1.31 \mathrm{~d}$ & $15.44 \mathrm{~d}$ & $15.50 \mathrm{e}$ \\
\hline goat manure + contr & & & $1.31 \mathrm{c}$ & $1.33 \mathrm{c}$ & $15.36 \mathrm{~d}$ & $15.46 \mathrm{e}$ \\
\hline control $1+$ plastic s & & & $1.29 \mathrm{~d}$ & $1.29 \mathrm{e}$ & $15.60 \mathrm{c}$ & $15.71 \mathrm{~d}$ \\
\hline compost + plastic sh & & & $1.08 \mathrm{f}$ & $1.04 \mathrm{~h}$ & $16.89 \mathrm{a}$ & $16.89 \mathrm{a}$ \\
\hline goat manure + plasti & sheet & & $1.09 \mathrm{f}$ & $1.05 \mathrm{~h}$ & $16.82 \mathrm{a}$ & $16.84 a$ \\
\hline control $1+$ rice straw & & & $1.35 \mathrm{~b}$ & $1.37 \mathrm{~b}$ & $14.44 \mathrm{e}$ & $14.64 \mathrm{f}$ \\
\hline compost + rice straw & & & $1.22 \mathrm{e}$ & $1.17 \mathrm{~g}$ & $16.62 \mathrm{~b}$ & $16.66 \mathrm{~b}$ \\
\hline goat manure + rice $s$ & & & $1.23 \mathrm{e}$ & $1.22 \mathrm{f}$ & $16.55 b$ & $16.59 \mathrm{c}$ \\
\hline Effect of drip irrig & ion systems, & rtilization anc & ing & & & \\
\hline drip irrigation & organic & cil mulbin & & & Vitam & $00 \mathrm{~g}$ \\
\hline systems & fertilization & Soll muicning & 2012 & 2013 & 2012 & 2013 \\
\hline & control 1 & control 2 & $1.48 \mathrm{~b}$ & $1.49 \mathrm{~b}$ & $14.22 \mathrm{j}$ & $14.32 \mathrm{~m}$ \\
\hline & control 1 & plastic sheet & $1.27 \mathrm{e}$ & $1.28 \mathrm{~g}$ & $15.87 \mathrm{e}$ & $15.88 \mathrm{~g}$ \\
\hline & control 1 & rice straw & $1.28 \mathrm{e}$ & $1.30 \mathrm{f}$ & $15.84 \mathrm{e}$ & $15.99 \mathrm{f}$ \\
\hline & compost & control 2 & $1.26 \mathrm{ef}$ & $1.25 \mathrm{~h}$ & $15.95 \mathrm{e}$ & $15.91 \mathrm{~g}$ \\
\hline sub surface & compost & plastic sheet & $1.07 \mathrm{k}$ & $1.00 \mathrm{~m}$ & $17.00 \mathrm{a}$ & $17.00 \mathrm{a}$ \\
\hline & compost & rice straw & 1.031 & $1.01 \mathrm{~m}$ & $16.88 \mathrm{ab}$ & $16.93 \mathrm{a}$ \\
\hline & goat manure & control 2 & $1.34 \mathrm{c}$ & $1.37 \mathrm{c}$ & $14.54 \mathrm{i}$ & $14.78 \mathrm{k}$ \\
\hline & goat manure & plastic sheet & $1.20 \mathrm{~h}$ & $1.11 \mathrm{k}$ & $16.72 \mathrm{c}$ & $16.72 b c$ \\
\hline & goat manure & rice straw & $1.21 \mathrm{~h}$ & $1.20 \mathrm{j}$ & $16.67 \mathrm{c}$ & $16.69 \mathrm{c}$ \\
\hline & control 1 & control 2 & $1.58 \mathrm{a}$ & $1.59 \mathrm{a}$ & $13.85 \mathrm{k}$ & $14.00 \mathrm{n}$ \\
\hline & control 1 & plastic sheet & $1.31 \mathrm{~d}$ & $1.34 \mathrm{~d}$ & $15.00 \mathrm{~g}$ & $15.11 \mathrm{i}$ \\
\hline & control 1 & rice straw & $1.34 \mathrm{c}$ & $1.36 \mathrm{~cd}$ & $14.87 \mathrm{~h}$ & $14.92 \mathrm{j}$ \\
\hline & compost & control 2 & $1.31 \mathrm{~d}$ & $1.32 \mathrm{e}$ & $15.25 \mathrm{f}$ & $15.51 \mathrm{~h}$ \\
\hline surface & compost & plastic sheet & $1.09 \mathrm{j}$ & 1.081 & $16.78 \mathrm{bc}$ & $16.79 \mathrm{~b}$ \\
\hline & compost & rice straw & $1.14 \mathrm{i}$ & 1.091 & $16.75 \mathrm{c}$ & $16.76 \mathrm{bc}$ \\
\hline & goat manure & control 2 & $1.35 \mathrm{c}$ & $1.37 \mathrm{c}$ & $14.34 \mathrm{j}$ & 14.511 \\
\hline & goat manure & plastic sheet & $1.23 \mathrm{~g}$ & $1.23 \mathrm{i}$ & $16.51 \mathrm{~d}$ & $16.61 \mathrm{~d}$ \\
\hline & goat manure & rice straw & $1.25 \mathrm{fg}$ & 1.24hi & $16.43 \mathrm{~d}$ & $16.50 \mathrm{e}$ \\
\hline
\end{tabular}

Means having the same letter (s) in each column or interaction are not significantly different at $5 \%$ level 


\section{Conclusion}

From the present study, it can be concluded that sub surface drip irrigation system with compost as fertilization and mulching the soil with plastic cover improved leaf area, leaf chlorophyll, number of leaves/shoot, shoot length, number of followers / shoot, fruit set \%, fruit length, fruit diameter, fruit weight, grains weight, TSS, total sugar and vitamin C in both seasons. On the other side, surface drip irrigation without any organic fertilization and soil mulching gave the highest total acidity as compared with all treatments used on Manfalouty cv. pomegranate cultivar at Baloza Experimental Station, Desert Research Center, North Sinai Governorate, Egypt.

\section{Reference}

[1]. A. O. A. C., (1985). Official methods of analysis. Association of Official Agricultural Chemists,14th ed: Benjamin Franklin station Washington, DC, USA, pp: 490-510.

[2]. Abd-ElaaI, A. H; Basma M. S. and Ghada Sh. Sh. (2007). Response of Superior Grapevines to Application of Filter Mud, Compost El-Nile and Green Manure J. Agric. Sd. Mansoura Univ., 32 (12): 10300 - 10309.

[3]. Abou El-Wafa, M. (2002). Effect of Some Treatments on Drought Resistance of Transplants of Some Pomegranate Cultivars. M.Sc. Thesis, Hort. Dept. Fac. Agric., Cairo Univ. Egypt, pp: 132.

[4]. Albert T.; KKarp, M. ; Starast and Paal T. (2010). The effect of mulching and pruning on the vegetative growth and yield of the half-high blueberry. Agron Res 8 (1): 759-769.

[5]. Arancon, N. Q.; Edward, C. A.; Atiyeh, R. M. and Metzger, J. D. (2004). Effect of vermicompost produced from cattle manure, food waste and paper waste on the growth and yield of peppers in the field. Pedobiologia, 49, 297- 306.

[6]. Bryla, D. R.; Trout, T. J. and Ayars, J. E. (2003). Growth and production of young peach trees irrigated by furrow, microjet, surface drip, or subsurface drip systems. Hort. Science, 38(6), pp.1112-1116.

[7]. Douh B.; Boujelben A.; Khila S. and Bel Haj Mguidiche A. (2013). Effect of Subsurface Drip Irrigation System Depth on Soil Water Content Distribution at Different Depths and Different Times after Irrigation. Larhyss Journal, 13, pp. 7-16.

[8]. Duncan, D. B. (1955). Multiple range and multiple F Test. Biometrics, 11: 1-42.

[9]. EI-Kosary S.; Bakr E. I.; Hussein I. A. and Sheren A. E. (2009). Effect of Soil Moisture Depletion and Mulching of Cv. Zaghloul Date Palm (Phoenix Dactylifera L.) Fruiting Under Desert Conditions. Mansoura University Journal of Agricultural Sciences, 34: 3, 3437-3452.

[10]. El Mardi, M. O.; Al Said, F. Al.; Sakit, C. B.; Al Kharusi, L. M.; Al Rahbi, I. N. and Al Mahrazi, K. (2007). Effect of pollination method, fertilizer and mulch treatments on the physical and chemical characteristics of date palm (Phoenix dactylifera L.). Fruit 1: Physical characteristics. Acta Horticulture 736: III International Date Palm Conference.

[11]. Hati K. M.; Mandal K. G.; Misra A. K, Gosh P. K. and Bandyopadhyay K. K. (2006). Effect of inorganic fertilizer and farmyard manure on soil physical properties, root distribution and water-use efficiency of soybean in Vertisols of central India. Bioresour. Technol. 16: $2182-2188$

[12]. Helweg, O. J. (1989). Evaluating the traveling trickle center pivot., ICID-Bulletin 38:1, 13-20.

[13]. Holland, D.; Hatib, K. and Bar-Yàakov, I., (2009). Pomegranate: Botany, horticulture, breeding. Horticultural Reviews 35, $127-$ 191.

[14]. Jalota S. K. and Prihar S. S. (1998). Reducing soil water evaporation with Tillage and Mulching. Iowa State University Press, Ames.IA.142P.

[15]. Janaki A. and Hkmsk H. (2009). Impact of Mulching on Soil Moisture, Plant Growth and Yield of Mauritius Pineapple (Ananas comosus. L. Merr) Journal of Food and Agriculture . Vol 2, No 1 15-21.

[16]. Kay, B. L. (1998). Mulching and chemical stabilizers for land reclamation in dry regions. pp 467-483. In. F. W. Schaller and P. sutten, editors. Reclamation of Drastically Distributed lands. Am. Soc. Agronomy Madison. WI.

[17]. Lu W.G.; Huang Q.W. and Shen Q. R. (2005). The effect of organic fertilizer and organic-inorganic fertilizer application on soil enzymes activities during watermelon growing period. Journal of Nanjing Agricultural. 28; 68-71

[18]. Misak R. F. and M. Y. Draz (1997)). Sand drift control of selected coastal and desert dunes in Egypt: case studies. Journal of Arid Environments 35: 17-28.

[19]. Monteith, (1973) In " principles of environmental physics". Edward Arnold, Ltd., London, U.K.

[20]. Munshower F. F. (1994). Practical hand book of disturbed land revegetation lewis. Publishers. Boca. Raton. FL.

[21]. Ramakrishna A. ;Tam H. M. ;Wani S. P. and Long T. D. (2006). Effect of mulch on soil temperature, moisture, weed infestation and yield of groundnut in northern Vietnam. Field Crop Res 95: 115-125.

[22]. Ren Z. G.; Chen Y. S. and Tang F. Q. (1996). Effect of inorganic fertilizer combined with organic manure on the micro flora and enzyme activities in paddy soil. Plant Nutrition and Fertilizer Science 2, 279-283.

[23]. Safar H. AL-Kahtani and Ahmed M. A. (2012). Effect of Different Mixtures of Organic Fertilizers on Vegetative Growth, Flowering, Fruiting and Leaf Mineral Content of Picual Olive Trees American-Eurasian J. Agric. \& Environ. Sci., 12 (8): $1105-$ 1112.

[24]. Stover, E. and Mercure, E. W. (2007). The Pomegranate: a new look at the fruit of paradise. HortScience 42, 1088-1092.

[25]. Sudadi Y. N.; Hidayati S. F. and Sumani M. N. (2007). Ketersediaan K dan hasil kedelai (Glycine max L. Cerril) pada tanah vertisol yang diberi mulsa dan pupuk kandang. J I Tanah Lingk 7 (1): 8-12 (in Indonesian).

[26]. Sun R. L.; Zhao B. Q. and Zhu L. S. (2003) Effects of long-term fertilization on soil enzyme activities and its role in adjustingcontrolling soil fertilit. Plant Nutrition and Fertilizer Science 9, 406-410.

[27]. Talat F. A.; Hashim N. H.; Abdul Razzaq G. and Sheikh A. A. (2012) Performance assessment of surface and subsurface drip irrigation system for date palm fruit trees. African Journal of Agricultural Research Vol. 7(10), pp. 1542-1549.

[28]. Tarara J. M. (2000) Micro climate modification with plastic mulch. Hort Science 35: 169-180

[29]. Tiwari K. N.; Mukesh K.; Santosh D. T.; Vikas K. S.; Maji M. K. and Karan A. K.(2014) Influence of Drip Irrigation and Plastic Mulch on Yield of Sapota (Achras zapota) and Soil Nutrients. Irrigate Drainage Sys Eng Volume 3: 1-8.

[30]. Zaman W. U.; Arshad M. and Saleem K. (2001). Distribution of nitrate -nitrogen in the soil profile under different irrigation methods. International, J. Agri. Biol., 2: 208-9. 